Article

\title{
Visible-broadband Localized Vector Vortex Beam Generator with a Multi-structure-composited Meta-surface
}

\author{
Zhuo Yang ${ }^{\circledR}$ and Dengfeng Kuang * \\ Tianjin Key Laboratory of Optoelectronic Sensor and Sensing Network Technology, and Institute of Modern \\ Optics, Nankai University, Tianjin 300350, China; nkyangzhuo@mail.nankai.edu.cn \\ * Correspondence: dfkuang@nankai.edu.cn; Tel.: +86-138-2086-8981
}

Received: 27 December 2018; Accepted: 24 January 2019; Published: 29 January 2019

check for updates

\begin{abstract}
We demonstrate a vortex beam generator meta-surface that consists of silver structures and graphene layers. The miniature material is just a few microns in size and the working part is only a few hundred nanometers thick. With the incidence of the linearly polarized beam, the meta-surface generates high-localized vector vortex beam with a high proportion of the longitudinal component. Being compared with the constituent part of the meta-surface, the multi-structure-combined meta-surface increases the localization by $250 \%$ and the longitudinal component proportion by $200 \%$. Moreover, the above artificial material can generate vortex beams in broadband within the visible light range. These novel optical properties have the potential to improve the precision and sensitivity of nanoparticle manipulation. The study serves as a foundation in optical miniaturization and integration, nanoparticle manipulation, high-efficiency optical and quantum communication, and light-driven micro-tools.
\end{abstract}

Keywords: vortex beam; meta-surface; optical manipulation

\section{Introduction}

Vector vortex beams that carry orbital angular momentum [1-4] are widely utilized in particle manipulation and separation [5-7], optical communication [8-11], quantum computing [12,13] and even light-driven micro-tools [14]. Formerly, vortex beams were tuned by spiral phase plates $[15,16]$, spatial light modulators $[17,18]$ and computer-generated holograms $[19,20]$. Nowadays, meta-surfaces [21-25] are utilized to generate optical fields for the integration and miniaturization of optical components. For instance, Pancharatnam-Berry phase planar elements [26-29] and plasmonic vortex lens [30-32] realize the function of converting circularly polarized beams into vortex beams. In recent years, combinations of spirals and tapers were proposed for generation of localized vortex beams [6,33-35]. In addition, designed artificial structures were reported to convert linearly polarized beams into localized vector vortex beams $[35,36]$. In other words, it was good for nano-miniaturization and integration of optical elements that localized vector vortex beams can be generated without extra polarizers. Nevertheless, the proportion of the longitudinal component and the localization of transmitted fields generated by meta-surfaces above is not high enough in theory $(<20 \%)$ [36], resulting in low-sensitivity and low-precision of particle manipulation, respectively. Besides, most vortex beam generators work only at several certain frequencies $[28,29,35]$. Therefore, broadband vortex beam generators are significantly useful in wider applications.

In this study, a vortex beam generator meta-surface is proposed. The multi-structure-composited meta-surface consists of silver structures and graphene layers. The miniature material is just a few microns in size and the working part is only a few hundred nanometers thick. With the incidence 
of the linearly polarized beam, the meta-surface generates high-localized vector vortex beam with a high proportion of the longitudinal component. Being compared with the constituent part of the meta-surface, the multi-structure-combined meta-surface increases the localization by $250 \%$ and the longitudinal component proportion by $200 \%$. Moreover, the above artificial material can generate vortex beams in broadband within the visible light range. These novel optical properties have the potential to improve the precision and sensitivity of nanoparticle manipulation. This study serves as a foundation in optical miniaturization and integration, nanoparticle manipulation, high-efficiency optical and quantum communication, and light-driven micro-tools.

\section{Materials and Methods}

The designed structure consists of a metallic dolphin-shaped cell circular array (MDCCA), metallic cylinder (MC) and graphene layer (GL). Simultaneously, ultrathin dielectric planar blocks are intended for substrates. In our study, materials of combinations are slightly different, including silver (in gray), graphene (in light purple) and silicon dioxide (in light blue) according to the legend that is inset of Figure 1. Dolphin-shaped cell meta-surface (DSCMS) is presented in Figure 1a. It consists of a MDCCA and dielectric substrate. Figure $1 b, d$,e show three types of combinations focused on in contemporary studies. Eight dolphin-shaped cells are angularly arranged to form MDCCAs, in the center of which the MCs are placed. The parameters $R_{1}$ and $R_{2}$ are defined as radii of the circular array and central metallic cylinder. The parameters $\mathrm{d}_{1}, \mathrm{~d}_{2}$ and $\mathrm{d}_{3}$ are defined as the thickness of substrate, metal and graphene. The GLs, which consists of several layers of graphene monolayer, are added to the position in the interlayer of metallic structures and dielectric substrate. Figure $1 \mathrm{~g}$ shows the geometric explanation of dolphin-shaped cell which is geometrically constituted by two inscribed cylinders of which radii are marked as $r_{1}$ and $r_{2}$ according to the Boolean operation. The dolphin-shaped cell can be defined as a surrounding area in two dimensions by two functions which are shown as follows:
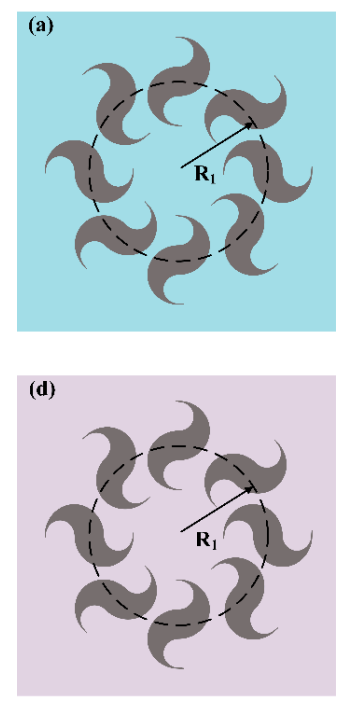
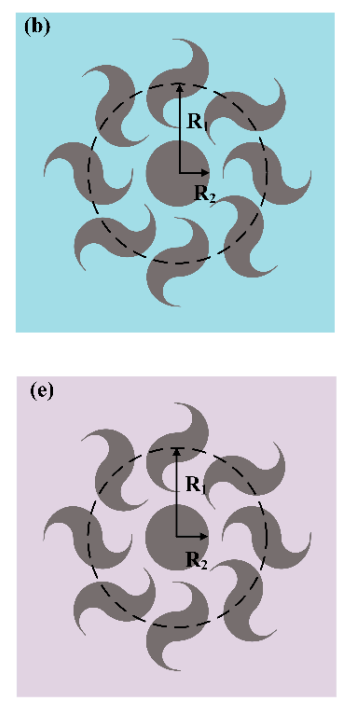
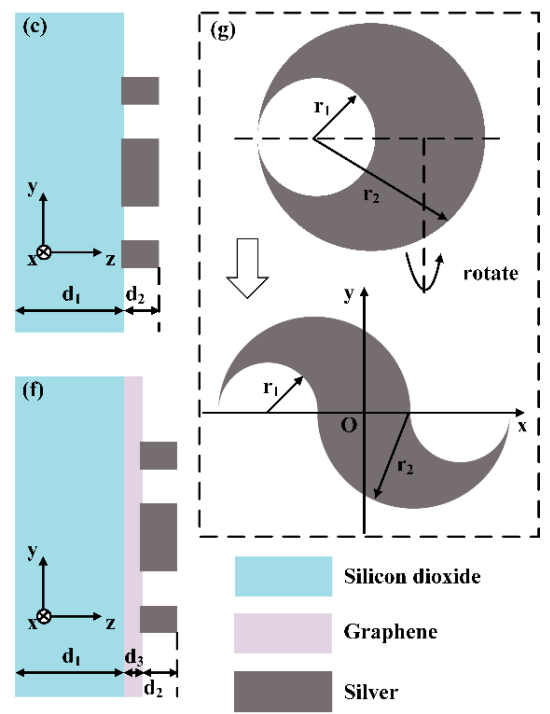

Figure 1. Schematic of the complex meta-surface which consists of metallic dolphin-shaped cell circular array (MDCCA), metallic cylinder (MC) and graphene layer (GL). (a) Schematic of dolphin-shaped cell meta-surface (DSCMS). (b) Schematic of combination of MDCCA and MC. (c) Lateral view of combination of MDCCA and MC. (d) Schematic of combination of MDCCA and GL. (e) Schematic of combination of MDCCA, MC and GL. (f) Lateral view of combination of MDCCA, MC and GL. (g) Geometric explanation of dolphin-shaped cell.

$$
f_{1}(x)= \begin{cases}\sqrt{\frac{3}{4} r_{2}^{2}-r_{2} x-x^{2}} & , x \in\left[-2 r_{1}-\frac{r_{2}}{2}, \frac{r_{2}}{2}\right) \\ -\sqrt{\left(2 r_{1}+r_{2}\right) x-x^{2}-r_{1} r_{2}-\frac{1}{4} r_{2}^{2}} & , x \in\left[\frac{r_{2}}{2}, 2 r_{1}+\frac{r_{2}}{2}\right]\end{cases}
$$




$$
f_{2}(x)= \begin{cases}\sqrt{-x^{2}-\left(2 r_{1}+r_{2}\right) x-r_{1} r_{2}-\frac{1}{4} r_{2}{ }^{2}} & , x \in\left[-2 r_{1}-\frac{r_{2}}{2},-\frac{r_{2}}{2}\right) \\ -\sqrt{\frac{3}{4} r_{2}^{2}+r_{2} x-x^{2}} & , x \in\left[-\frac{r_{2}}{2}, 2 r_{1}+\frac{r_{2}}{2}\right]\end{cases}
$$

The structural parameters of combinations are initialized as follows: $R_{1}=1000 \mathrm{~nm}, R_{2}=500 \mathrm{~nm}$, $r_{1}=150 \mathrm{~nm}, r_{2}=300 \mathrm{~nm}, d_{2}=100 \mathrm{~nm}, d_{3}=35 \mathrm{~nm}$. Three-dimensional finite-difference time domain (3D-FDTD) methods are used in simulations. Perfectly matched layers are utilized as simulation boundaries with the incident linearly polarized beams of which the wavelength is $660 \mathrm{~nm}$. Our own FDTD programs run with MATLAB 2013A (Nankai University, Tianjin, China). All of our simulations were performed on a PC machine (Nankai University, Tianjin, China) configured with an Intel 8-Core i7-7700 CPU @ 3.60 GHz (Nankai University, Tianjin, China).

\section{Results and Discussions}

Figure 2 shows the phase distribution of the longitudinal transmission fields which are generated by the four types of nano-structures shown in Figure $1 \mathrm{a}, \mathrm{b}, \mathrm{d}$,e. It is obviously seen that all four nano-optical devices can generate vortex phases. However, there is a noteworthy difference in the radii of the vortexes at the same propagation distance. The incident beam passes through the central metal structure and the underlying graphene, causing a phase delay at the center, thereby causing a significant phase change at the edge of the central vortex field, which is significantly related to the localization of vortex beams. The characters above are illustrated in Figure 3. According to Formula A2, the phase delays are different as the beam passes through air and metal, causing a significant phase change shown in Figure 3. Figure 4a,c shows the amplitude of the optical field generated by a single metallic dolphin-shaped element to discuss the excitation process of the longitudinal field. The intensity of the incident light field is normalized. Surface plasmon polaritons (SPPs) were excited at the boundary of the metallic cells, which is explained by Formula A1 (Appendix A), then guided by the metallic element and finally squeezed to the tips to form highly localized strong electromagnetic fields of which the enhancement factor were greater than $2^{15}$. The strong fields radiated from the tips, then the vortex longitudinal field was generated.
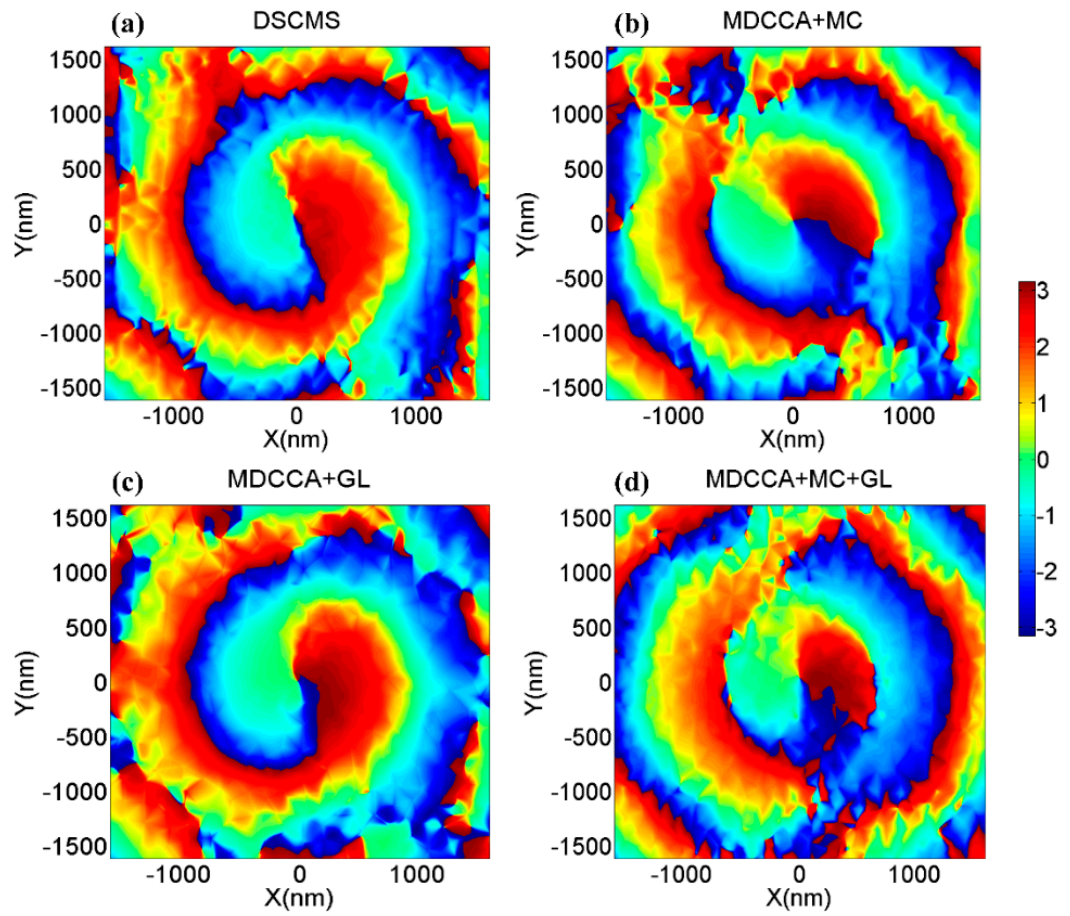

Figure 2. The phase distributions of the transmission fields of $\mathrm{z}$ components which are generated by (a) DSCMS, (b) combination of MDCCA and MC, (c) combination of MDCCA and GL, (d) combination of MDCCA, MC and GL at the distances $D=2000 \mathrm{~nm}$. 

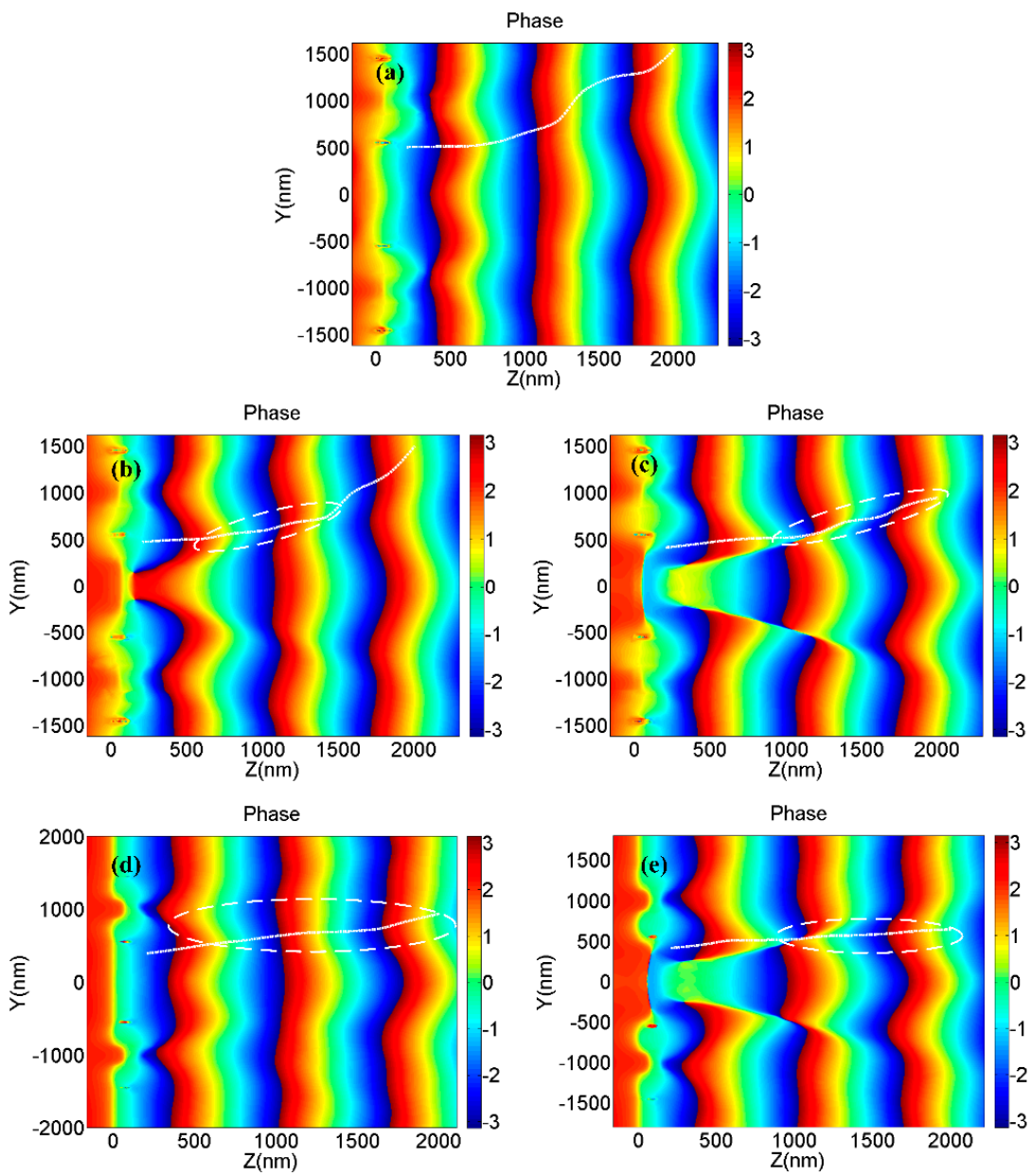

Figure 3. The phase distributions of the transmission fields generated by (a) DSCMS and combination of (b) MDCCA and MC ( $\left.R_{2}=300 \mathrm{~nm}\right),(\mathbf{c})$ MDCCA and MC $\left(R_{2}=500 \mathrm{~nm}\right)$, (d) MDCCA and GL, (e) MDCCA, MC $\left(R_{2}=500 \mathrm{~nm}\right)$ and GL at the plane $x=0$, where the white solid lines are the edges of vortex phases and the white dashed lines indicate the coincident regions of vortex phase edges and phase mutations.

Taking the simulation of the combination of MDCCA and MC as an example for characters of vortex beams, we kept the radius of circularly array fixed $\left(R_{1}=1000 \mathrm{~nm}\right)$ and varied the radius of MC from 0 to $500 \mathrm{~nm}$ with the step length of $100 \mathrm{~nm}$. Figure 5a shows the radii of vortex phases (RVPs) generated by combinations of MDCCA and MC with different $\mathrm{R}_{2}$ and the same incident wavelength. For instance, at the distance $D=1000 \mathrm{~nm}$ the RVP stood at approximately $652 \mathrm{~nm}$ without the MC, $648 \mathrm{~nm}$ with $R_{2}=100 \mathrm{~nm}, 612 \mathrm{~nm}$ with $R_{2}=200 \mathrm{~nm}, 593 \mathrm{~nm}$ with $R_{2}=300 \mathrm{~nm}, 588 \mathrm{~nm}$ with $R_{2}=400 \mathrm{~nm}$ and only $518 \mathrm{~nm}$ with $R_{2}=500 \mathrm{~nm}$. Therefore, the radius of MC plays a positive role in the localization of vortex phase generated by combination meta-surface. At its best, a MC roughly doubles the localization of the vector vortex beam. The localization of the vector vortex beam was significantly improved with $R_{2}=500 \mathrm{~nm}$, but the localization decreased rapidly when the propagation distance was greater than $1800 \mathrm{~nm}$. Similarly, Figure $5 \mathrm{~b}$ shows the proportion of the longitudinal component of the transmitted field (PLT) generated by combinations of MDCCA and MC with different $R_{2}$ and the same incident wavelength of $660 \mathrm{~nm}$. As is shown in the line graph, among the six combinations with different $R_{2}$, the PLT with $R_{2}=500 \mathrm{~nm}$ is the highest and that without the MC is the lowest at the distance below $2000 \mathrm{~nm}$. This phenomenon is particularly evident when the propagation distance is between $1000 \mathrm{~nm}$ and $1600 \mathrm{~nm}$. It can be seen that the radius of MC plays a positive role in the proportion of the longitudinal component of the transmitted field. At best, the PLT can be increased to about three times its value. When the particle is placed in the localized 
vortex field, the range of interaction between the particle and the light field decreased due to the reduction of the vortex area. Since the micro-optical device, such as the meta-surface, can precisely control the light field in sub-wavelength region and manipulate the particles placed in localized light field, the above-mentioned meta-surface with $R_{2}=500 \mathrm{~nm}$ exciting localized vector vortex beam can significantly improve the precision of particle manipulation. Moreover, the high proportion of the longitudinal component of transmitted fields can be obtained by the device above with $R_{2}=500 \mathrm{~nm}$ for ideal manipulation sensitivity because particle manipulation is closely related to the longitudinal component of vector optical fields. Figure $5 \mathrm{c}$ shows the RVPs utilizing different combinations with the same incident wavelength. At the distance $D=1400 \mathrm{~nm}$, for instance, the RVP stood at approximately $690 \mathrm{~nm}$ with the combinations of MDCCA and GL, which was greater than that with the combination of MDCCA and MC $\left(R_{2}=500 \mathrm{~nm}\right)$. However, at the distance $D=2000 \mathrm{~nm}$, the RVP stood at about $935 \mathrm{~nm}$ with the combination of MDCCA and GL, which was less than that with the combination of MDCCA and MC $\left(R_{2}=500 \mathrm{~nm}\right)$. This shows that the GL has a higher local potential for the vortex field at a further distance of propagation than the MC. In other words, using the GL as a part of the meta-surface above will result in greater particle manipulation accuracy at longer distances. At the distance $D>1300 \mathrm{~nm}$, the RVPs with the combination of MDCCA, MC $\left(R_{2}=500 \mathrm{~nm}\right)$ and GL were less than that with the combination of MDCCA and one or two of the MC and GL, which means that the combinations of the three elements can produce highly localized vector vortex beams over a relatively long propagation distance. Figure $5 \mathrm{~d}$ shows the PLTs when using different structures at different distances. The PLT through the GL was smaller than that without GL, which is related to the relatively high absorption of the graphene. At best, the GL increased localization by approximately $50 \%$. Compared to individual devices, the combination of MDCCA, MC $\left(R_{2}=500 \mathrm{~nm}\right)$ and GL could increase localization by $250 \%$ and PLT by $200 \%$. The binding of graphene layers to metals can improve localized resonances, which can improve the localization and the longitudinal component proportion simultaneously. When composite structures composed of three kinds of elements are used for particle manipulation, precision and sensitivity can be taken into account, which provides a foundation in optical elements design to build miniaturized and integrated optical systems of high-precision and high-sensitivity macromolecule manipulation.
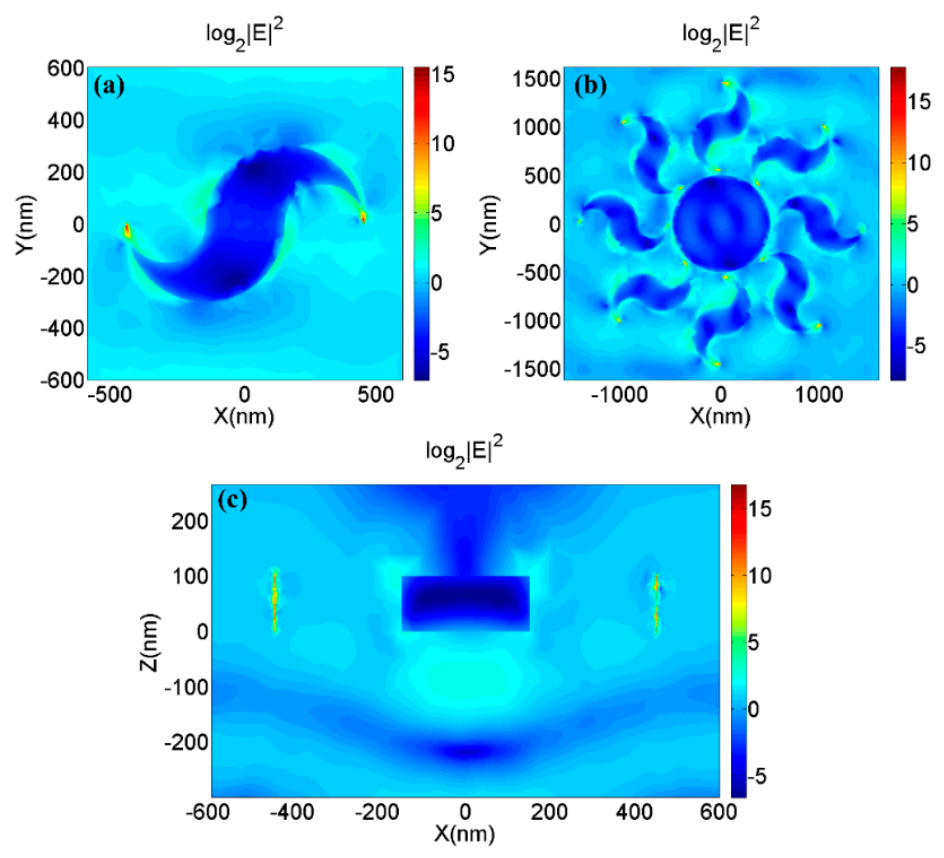

Figure 4. (a) The amplitude of optical field generated by a single metallic dolphin-shaped element at $z=100 \mathrm{~nm}$ and (b) that of optical field generated by the combination of MDCCA and MC. (c) The amplitude of optical field generated by a single metallic dolphin-shaped element at $y=0$. 

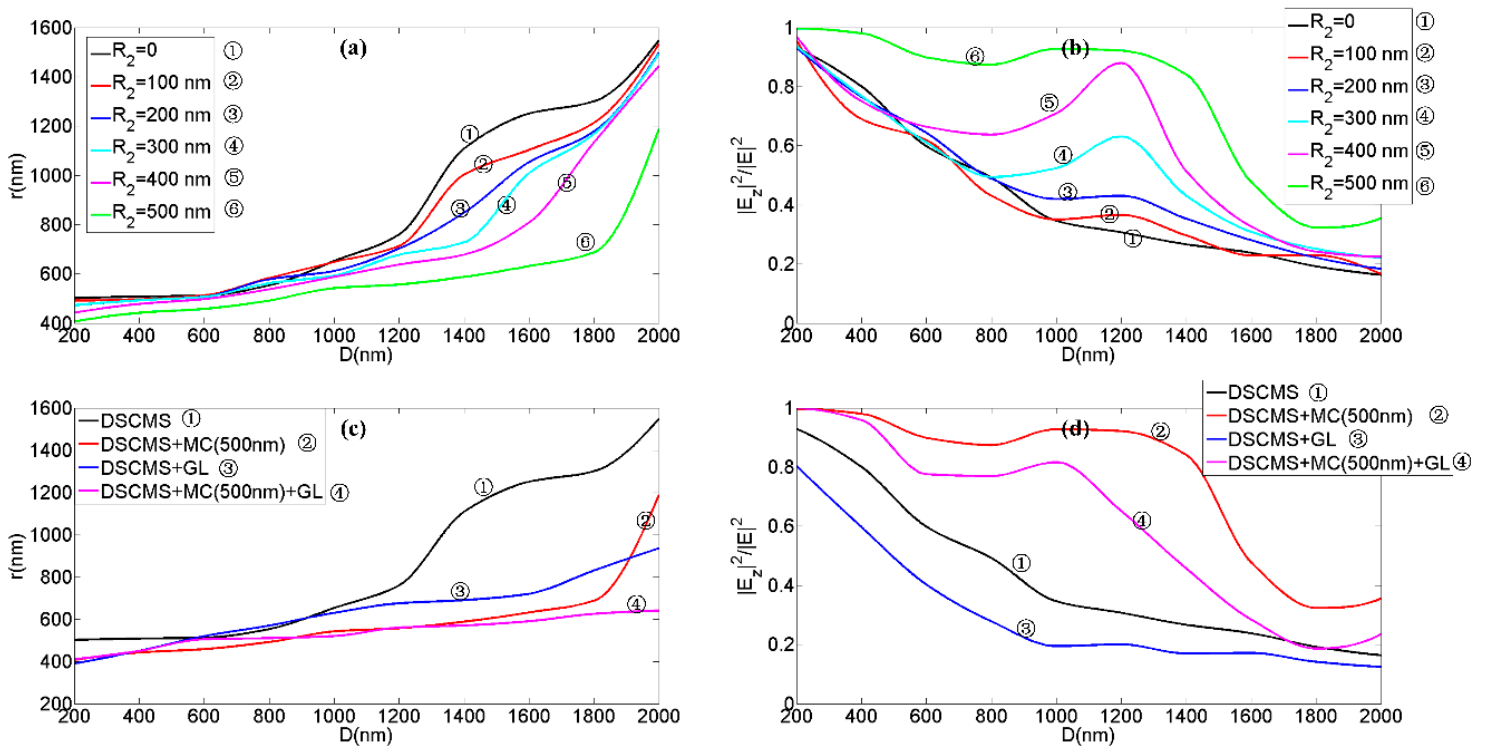

Figure 5. (a) The radii of transmitted vortex phases of the light fields generated by the combination of MDCCA and MC at different distances. (b) The proportion of the longitudinal component of the transmitted field at different distances when using the combination of MDCCA and MC.

(c) The radii of transmitted vortex phases generated by different structures at different distances.

(d) The proportion of the longitudinal component of the transmitted field when using different structures at different distances.

We also study the vortex beam excitation characteristics of the composite devices in visible light region in order to expand the application in the wide-band wave-length range. Figure 6a shows the transmission spectrum of the combination of MDCCA and MC with $R_{2}=500 \mathrm{~nm}$. On the whole, the transmittance was lower in the wavelength range of $600 \mathrm{~nm}$ and $650 \mathrm{~nm}$ than that in the other visible range. However, it will not affect the vortex beam generation because high-intensity micro-focus incident light can compensate for the loss caused by the low transmittance of the devices. In contrast, the PLTs excited by the devices in visible broadband directly determined whether the device can be applied for high-efficiency excitation of the localized vector vortex beam in visible light. Figure $6 \mathrm{~b}$ shows the PLTs when using the combination of MDCCA and MC $\left(R_{2}=500 \mathrm{~nm}\right)$ in the incident wavelength range from $500 \mathrm{~nm}$ to $750 \mathrm{~nm}$ at different distances. The areas with a reddish color indicate that the PLTs were greater at the corresponding wavelength and propagation distance. The longitudinal component proportions were less than $70 \%$ at the region close to $550 \mathrm{~nm}$. We hold the view that the proportion of light fields that are not regulated is larger because of the higher transmittance, causing the PVLs with smaller values. The device relies on transmitted excited fields to generate vortex beams. Unlike other common vortex beam generators, the device had very little limitation in the excitation wavelength band. It confirms that the devices can produce localized vector vortex beam in broad-band, which provides the guidance for the excitation and utilization of localized vector vortex light beams generated by the complex meta-surfaces above. 

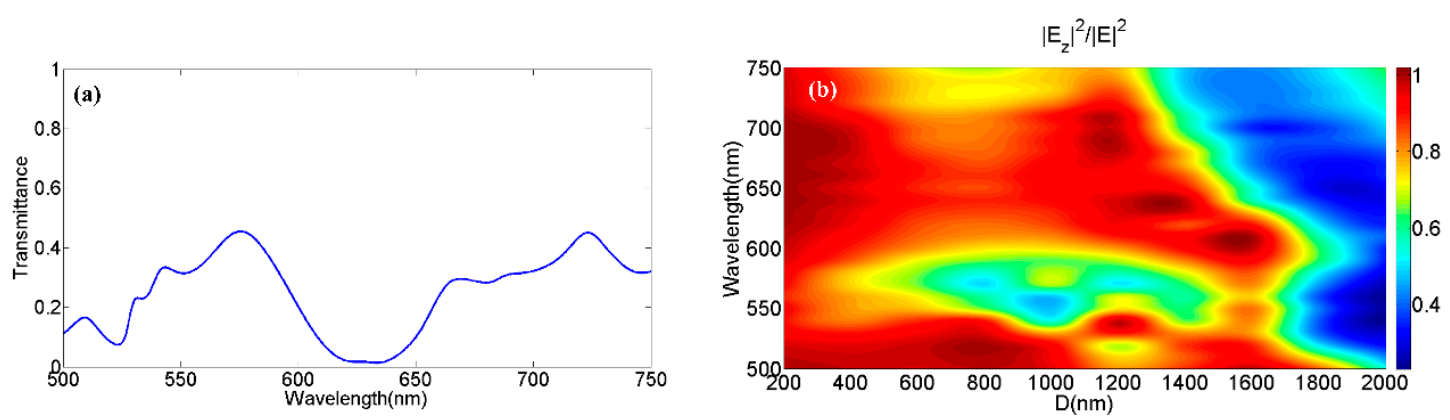

Figure 6. (a) Transmission spectrum of the combination of MDCCA and MC $\left(R_{2}=500 \mathrm{~nm}\right)$. (b) The PLTs when using the combination of MDCCA and MC $\left(R_{2}=500 \mathrm{~nm}\right)$ in the incident wavelength range from $500 \mathrm{~nm}$ to $750 \mathrm{~nm}$ at different distances.

\section{Conclusions}

We demonstrate a vortex beam generator meta-surface that consists of silver structures and graphene layers. The advantages of the artificial material are as follows:

- The miniature material is just a few microns in size and the working part is only a few hundred nanometers thick. The side-length of the working part is less than $1500 \mathrm{~nm}$. Moreover, the working part is approximately $135 \mathrm{~nm}$ thick.

- With the incidence of the linearly polarized beam, the meta-surface generates high-localized vector vortex beam with a high proportion of the longitudinal component. Being compared with the constituent part of the meta-surface, the multi-structure-combined meta-surface increases the localization by $250 \%$ and the longitudinal component proportion by $200 \%$.

- The material can generate vortex beams in broadband within the visible light range. The results confirm that the devices can produce localized vector vortex beam in broadband, which provides the guidance for the excitation and utilization of localized vector vortex light beams generated by complex meta-surfaces above.

- Those above novel optical properties have the significantly potential to improve the precision and sensitivity of nanoparticle manipulation. The study serves as a foundation in optical miniaturization and integration, nanoparticle manipulation, high-efficiency optical and quantum communication, and light-driven micro-tools.

Author Contributions: Z.Y. carried out the concrete simulations, did mathematical work and wrote the manuscript. D.K. read and revised the manuscript.

Funding: This research was funded by Natural Science Foundation of Tianjin City, grant number 18JCZDJC38200. This research was also funded by National Natural Science Foundation of China (NSFC), grant number 11274186.

Acknowledgments: This research was supported by Engineering Research Center of Thin Film Photo-electronics Technology, Ministry of Education, 111 Project (B16027) and International Cooperation Base for New PV Technology.

Conflicts of Interest: The authors declare no conflict of interest.

\section{Appendix A}

SPPs propagate along the surface of the metallic structure with a wavelength shown as follows:

$$
\lambda_{S P P}=\lambda_{0} \sqrt{\frac{\varepsilon_{m}^{\prime}+\varepsilon_{d}}{\varepsilon_{m}^{\prime} \varepsilon_{d}}}
$$

where $\varepsilon_{m}^{\prime}$ is the real part of the relative permittivity. When the wavelength of the incident field $\lambda_{0}$ is $660 \mathrm{~nm}$, the wavelength of SPPs $\lambda_{S P P}$ is approximately $643 \mathrm{~nm}$, confirming the SPPs bound to the metal surface. SPPs are excited at the boundary of the metallic cells, then guided by the metallic element and 
finally squeezed to the tips to form highly localized strong electromagnetic fields. The strong fields radiate from the tips, then the vortex longitudinal field is generated.

The transmission phase of the planar optical element can be directly obtained as follows [37]:

$$
\phi=\arg \left(\frac{2 Y_{o}-Y_{e}}{2 Y_{o}+Y_{e}}+1\right)
$$

where

$$
\begin{gathered}
Y_{0}= \begin{cases}\frac{\sqrt{\varepsilon_{0} \mu_{0} k_{0}^{2}-k_{x}^{2}}}{\mu_{0} \omega} & \text { for TE } \\
\frac{\varepsilon_{0} \omega}{\sqrt{\varepsilon_{0} \mu_{0} k_{0}^{2}-k_{x}^{2}}} & \text { for TM }\end{cases} \\
Y_{e}=-i \omega h \frac{\sum_{j=1}^{n} D_{z j} V_{j}}{\sum_{j=1}^{n} E_{z j} V_{j}}
\end{gathered}
$$

It can be seen from the formulas that the phase difference generated by different materials is different. The above properties determine the localization of the transmitted fields.

With the incident linearly polarized beam, the transmission of the incident light from the structure can be obtained from:

$$
T=\tau T_{1}+(1-\tau) T_{2}
$$

where

$$
\begin{gathered}
T_{1}=\frac{4 \zeta_{A} \zeta_{S}}{\left(\zeta_{0} B+C\right)\left(\zeta_{0} B+C\right)^{*}} \\
T_{2}=\frac{2 \zeta_{A}}{\zeta_{A}+\zeta_{G}} \frac{\cos \theta_{i}}{\cos \theta_{t}} \\
\tau=\frac{\pi\left(R_{2}^{2}-8 r_{1}^{2}+8 r_{2}^{2}\right)}{S^{2}}
\end{gathered}
$$

Here, $S$ is the length of the side of the meta-surface. For the combination of MDCCA and MC, the parameters $B$ and $C$ can be obtained from:

$$
\left[\begin{array}{l}
B \\
C
\end{array}\right]=M\left[\begin{array}{c}
1 \\
\zeta_{G}
\end{array}\right]
$$

where

$$
M=\left[\begin{array}{cc}
\cos \delta_{M} & \frac{i}{\zeta_{M}} \sin \delta_{M} \\
i \zeta_{M} \sin \delta_{M} & \cos \delta_{M}
\end{array}\right]
$$

and

$$
\begin{gathered}
\zeta_{j=(A, M, G)}=\frac{n_{j \prime=(\text { air }, \text { metal }, \text { substrate })}}{\cos \theta_{i}} \\
\delta_{M}=\frac{2 \pi}{\lambda} n_{\text {metal }} d_{2} \cos \theta_{i}
\end{gathered}
$$

\section{References}

1. Gao, H.; Li, Y.; Chen, L.; Jin, J.; Pu, M.; Li, X.; Gao, P.; Wang, C.; Luo, X.; Hong, M. Quasi-Talbot effect of orbital angular momentum beams for generation of optical vortex arrays by multiplexing metasurface design. Nanoscale 2018, 10, 666-671. [CrossRef]

2. Li, S.; Shen, B.; Zhang, X.; Bu, Z.; Gong, W. Conservation of orbital angular momentum for high harmonic generation of fractional vortex beams. Opt. Express 2018, 26, 23460-23470. [CrossRef] [PubMed] 
3. Jiang, S.; Chen, C.; Zhang, H.; Chen, W. Achromatic electromagnetic metasurface for generating a vortex wave with orbital angular momentum (OAM). Opt. Express 2018, 26, 6466-6477. [CrossRef]

4. Devlin, R.C.; Ambrosio, A.; Rubin, N.A.; Mueller, J.B.; Capasso, F. Arbitrary spin-to-orbital angular momentum conversion of light. Science 2017, 358, 896-901. [CrossRef] [PubMed]

5. Yeom, J.; Yeom, B.; Chan, H.; Smith, K.W.; Dominguez-Medina, S.; Bahng, J.H.; Zhao, G.; Chang, W.S.; Chang, S.J.; Chuvilin, A. Chiral templating of self-assembling nanostructures by circularly polarized light. Nat. Mater. 2014, 14, 66-72. [CrossRef] [PubMed]

6. Yang, Z.; Saleh, A.A.E.; Haar, M.A.V.D.; Baum, B.; Briggs, J.A.; Lay, A.; Reyesbecerra, O.A.; Dionne, J.A. Nanoscopic control and quantification of enantioselective optical forces. Nat. Nanotechnol. 2017, 12, 1055-1060.

7. Syubaev, S.; Zhizhchenko, A.; Kuchmizhak, A.; Porfirev, A.; Pustovalov, E.; Vitrik, O.; Kulchin, Y.; Khonina, S.; Kudryashov, S. Direct laser printing of chiral plasmonic nanojets by vortex beams. Opt. Express 2017, 25, 10214-10223. [CrossRef] [PubMed]

8. Wang, J. Advances in communications using optical vortices. Photon. Res. 2016, 4, B14-B28. [CrossRef]

9. Ndagano, B.; Nape, I.; Cox, M.A.; Rosales-Guzman, C.; Forbes, A. Creation and detection of vector vortex modes for classical and quantum communication. J. Lightwave Technol. 2018, 36, 292-301. [CrossRef]

10. Han, Y.; Liu, Y.G.; Wang, Z.; Huang, W.; Chen, L.; Zhang, H.W.; Yang, K. Controllable all-fiber generation/conversion of circularly polarized orbital angular momentum beams using long period fiber gratings. Nanophotonics 2018, 7, 287-293. [CrossRef]

11. Yan, X.; Guo, L.; Cheng, M.; Li, J. Controlling abruptly autofocusing vortex beams to mitigate crosstalk and vortex splitting in free-space optical communication. Opt. Express 2018, 26, 12605-12619. [CrossRef]

12. Ding, D.S.; Zhang, W.; Zhou, Z.Y.; Shi, S.; Xiang, G.Y.; Wang, X.S.; Jiang, Y.K.; Shi, B.S.; Guo, G.C. Quantum storage of orbital angular momentum entanglement in an atomic ensemble. Phys. Rev. Lett. 2015, 114, 050502. [CrossRef] [PubMed]

13. Nicolas, A.; Veissier, L.; Giner, L.; Giacobino, E.; Maxein, D.; Laurat, J. A quantum memory for orbital angular momentum photonic qubits. Nat. Photon. 2014, 8, 234-238. [CrossRef]

14. Villangca, M.J.; Palima, D.; Bañas, A.R.; Glückstad, J. Light-driven micro-tool equipped with a syringe function. Light Sci. Appl. 2016, 5, e16148. [CrossRef] [PubMed]

15. Ruffato, G.; Massari, M.; Romanato, F. Generation of high-order Laguerre-Gaussian modes by means of spiral phase plates. Opt. Lett. 2014, 39, 5094-5097. [CrossRef]

16. Xin, J.; Dai, K.; Zhong, L.; Na, Q.; Gao, C. Generation of optical vortices by using spiral phase plates made of polarization dependent devices. Opt. Lett. 2014, 39, 1984-1987. [CrossRef]

17. Forbes, A.; Dudley, A.; Mclaren, M. Creation and detection of optical modes with spatial light modulators. Adv. Opt. Photon. 2016, 8, 200-227. [CrossRef]

18. Forbes, A.; Rosalesguzmán, C.; Bhebhe, N. Simultaneous generation of multiple vector beams on a single SLM. Opt. Express 2017, 25, 25697-25706.

19. Guillon, M.; Forget, B.C.; Foust, A.J.; De, S.V.; Ritschmarte, M.; Emiliani, V. Vortex-free phase profiles for uniform patterning with computer-generated holography. Opt. Express 2017, 25, 12640-12652. [CrossRef]

20. Ronzitti, E.; Ventalon, C.; Canepari, M.; Forget, B.C.; Papagiakoumou, E.; Emiliani, V. Recent advances in patterned photostimulation for optogenetics. J. Opt. 2017, 19, 113001. [CrossRef]

21. Shi, F.; Qiu, M.; Zhang, L.; Lam, E.Y.; Lei, D.Y. Multi-plane Illumination Enabled by Fourier-Transform Metasurfaces for High-Speed Light-Sheet Microscopy. ACS Photonics 2018, 5, 1676-1684. [CrossRef]

22. Shen, F.; Kang, Q.; Wang, J.; Guo, K.; Zhou, Q.; Guo, Z. Dielectric Metasurface-Based High-Efficiency Mid-Infrared Optical Filter. Nanomaterials 2018, 8, 938. [CrossRef] [PubMed]

23. Chen, J.; Wang, K.; Long, H.; Han, X.; Hu, H.; Liu, W.; Wang, B.; Lu, P. Tungsten Disulfide-Gold Nanohole Hybrid Metasurfaces for Nonlinear Metalenses in the Visible Region. Nano Lett. 2018, 18, 1344-1350. [CrossRef] [PubMed]

24. Guo, Z.; Xu, H.; Guo, K.; Shen, F.; Zhou, H.; Zhou, Q.; Gao, J.; Yin, Z. High-Efficiency Visible Transmitting Polarizations Devices Based on the GaN Metasurface. Nanomaterials 2018, 8, 333. [CrossRef] [PubMed]

25. Shibanuma, T.; Maier, S.A.; Albella, P. Polarization control of high transmission/reflection switching by all-dielectric metasurfaces. Appl. Phys. Lett. 2018, 112, 063103. [CrossRef]

26. Lin, D.; Fan, P.; Hasman, E.; Brongersma, M.L. Dielectric gradient metasurface optical elements. Science 2014, 345, 298-302. [CrossRef] [PubMed] 
27. Yue, F.; Wen, D.; Xin, J.; Gerardot, B.D.; Li, J.; Chen, X. Vector Vortex Beam Generation with a Single Plasmonic Metasurface. ACS Photonics 2016, 3, 1558-1563. [CrossRef]

28. Liu, Y.; Ke, Y.; Zhou, J.; Liu, Y.; Luo, H.; Wen, S.; Fan, D. Generation of perfect vortex and vector beams based on Pancharatnam-Berry phase elements. Sci. Rep. 2017, 7, 44096. [CrossRef]

29. Wintz, D.; Ambrosio, A.; Zhu, A.Y.; Genevet, P.; Capasso, F. Anisotropic surface plasmon polariton generation using bimodal V-antenna based metastructures. ACS Photonics 2016, 4, 22-27. [CrossRef]

30. Kim, H.; Park, J.; Cho, S.W.; Lee, S.Y.; Kang, M.; Lee, B. Synthesis and dynamic switching of surface plasmon vortices with plasmonic vortex lens. Nano Lett. 2010, 10, 529-536. [CrossRef]

31. Zilio, P.; Parisi, G.; Garoli, D.; Carli, M.; Romanato, F. Bi-layer holey plasmonic vortex lenses for the far field transmission of pure orbital angular momentum light states. Opt. Lett. 2014, 39, 4899-4902. [CrossRef] [PubMed]

32. Chen, C.F.; Ku, C.T.; Tai, Y.H.; Wei, P.K.; Lin, H.N.; Huang, C.B. Creating Optical Near-Field Orbital Angular Momentum in a Gold Metasurface. Nano Lett. 2015, 15, 2746-2750. [CrossRef]

33. Li, J.; Mu, J.; Wang, B.; Ding, W.; Liu, J.; Guo, H.; Li, W.; Gu, C.; Li, Z.Y. Direct laser writing of symmetry-broken spiral tapers for polarization-insensitive three-dimensional plasmonic focusing. Laser Photon. Rev. 2014, 8, 602-609. [CrossRef]

34. Garoli, D.; Zilio, P.; Gorodetski, Y.; Tantussi, F.; De Angelis, F. Beaming of helical light from plasmonic vortices via adiabatically tapered nanotip. Nano Lett. 2016, 16, 6636-6643. [CrossRef]

35. Cheng, F.; Kuang, D.F.; Dong, L.Q.; Cao, Y.Y.; Gu, P.C. Tuning of nanofocused vector vortex beam of metallic granary-shaped nanotip with spin-dependent dielectric helical cone. Opt. Express 2017, 25, 17393-17401. [CrossRef] [PubMed]

36. Yang, Z.; Kuang, D.F.; Cheng, F. Vector vortex beam generation with dolphin-shaped cell meta-surface. Opt. Express 2017, 25, 22780-22788. [CrossRef] [PubMed]

37. Luo, X.G. Principles of electromagnetic waves in metasurfaces. Sci. China Phys. Mech. Astron. 2015, 58, 594201. [CrossRef]

(C) 2019 by the authors. Licensee MDPI, Basel, Switzerland. This article is an open access article distributed under the terms and conditions of the Creative Commons Attribution (CC BY) license (http://creativecommons.org/licenses/by/4.0/). 